\title{
Enhanced Site specific Preparation of SEM Cross Sections and TEM Samples by using CrossBeam Technology
}

\author{
P. Gnauck, P. Hoffrogge, M. Schumann, G. Bauhammer
}

LEO Elektronenmikroskopie GmbH, Oberkochen, Germany

In recent years focused ion beam (FIB) systems were routinely used for site specific cross sectioning and sample preparation for the transmission electron microscope (TEM). A wide range of different materials such as metals, semiconductors, ceramics and even biological substances can be covered. By combining the technique of focused ion beam and field emission SEM into an integrated CrossBeam tool the accuracy of TEM sample preparation and cross sectioning is significantly extended. In this article we report on improvements made in SEM cross sectioning and TEM sample preparation by using the CrossBeam technology.

The standard FIB cross sectioning is basically a blind process. The sample surface is imaged with the FIB before cutting to determine the area of interest. After this the sample is milled and polished with predefined milling patterns. Without the possibility of monitoring the milling process directly the area of interest is easily destroyed.

The capability of CrossBeam tools to image the sample in real time at high resolution during the ion milling process enables the operator to perform extremely accurate site specific cross sections. The milling and polishing process can be stopped exactly at the detail of interest e.g. in the very center of a tungsten contact plug in a semiconductor device (FIG.1). Especially in the case of TEM sample preparation the danger of destroying the fine lamella is reduced to a minimum.

Another advantage of the CrossBeam technology is the time saving cut and see operation. The sample is imaged during or immediately after the polishing. This leads to extremely short inspection times for each cross section.

Several TEM sample preparation techniques using FIB, such as pre-thinning and lift-out techniques have been published [1-3]. The FIB lift-out technique allows thin membranes to be extracted from bulk material, which saves a lot of sample pre-thinning time and is very successful in the preparation of site specific cross sections and planar samples.

However TEM sample preparation can be automated by using scripts and macros the best accuracy is achieved if the milling is done manually with direct SEM observation. In a first step the sample is milled and polished from the front side under continuous SEM control until the detail of interest is visible. In the second step the sample is rotated by $180^{\circ}$ and the backside of the sample is milled and polished under continuous SEM control until the desired thickness is achieved (FIG. 2). By imaging the TEM sample in the SEM the danger of destroying the TEM lamella due to drift etc. is minimized. Another opportunity of the direct SEM imaging is a very straight control of the specimen thickness and electron transparency during the ion milling process.

The best compromise concerning time and accuracy is achieved if different samples are pre-thinned automatically overnight to a thickness of about $1 \mu \mathrm{m}$ and then polished manually under high resolution SEM observation. 


\section{References}

[1] L. R. Herlinger, S. Chevacharoenkul, D. C. Erwin, ISTFA 1996, p415

[2] R. Rai, S. Subramanian, S. Rose, J. Conner, P. Schani, J. Moss, ISTFA 2000, p415

[3] T. L. Shofner, J. L. Drown, S. R. Brown, B. B. Rossie, M. A. Decker, Y. S. Obeng, F. A. Stevie, ISTFA 2000, p459

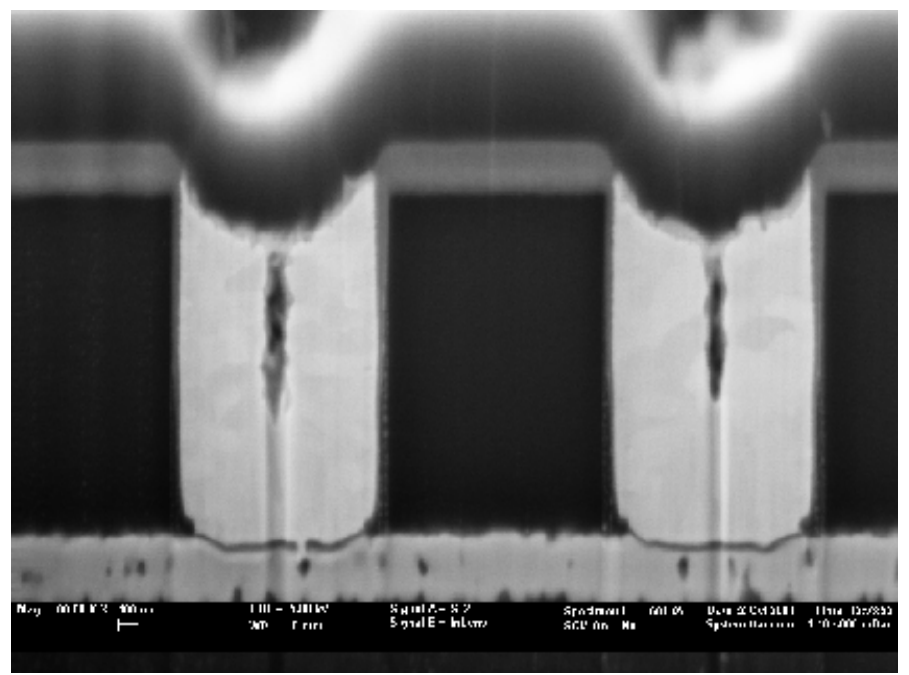

FIG. 1: Cross section through tungsten plugs in a semiconductor device. The milling process was stopped exactly in the centre of the plugs.
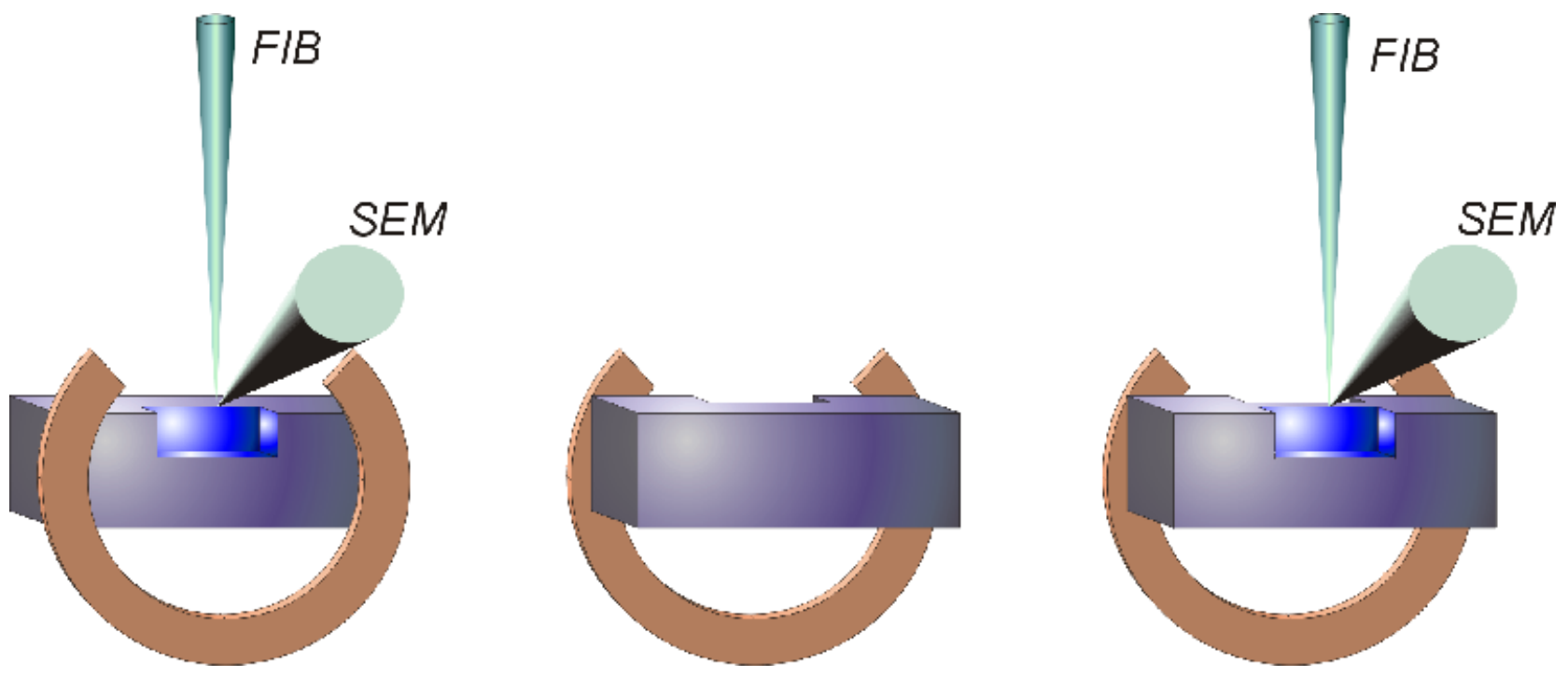

FIG. 2: Steps for TEM sample preparation using the CrossBeam technology. In step 1 the sample is milled and polished from the first side under continuous SEM control. In the second step the sample is rotated by $180^{\circ}$ and the backside of the sample is milled and polished under continuous SEM control until the desired thickness is achieved. 